\title{
A catalyst for transforming health systems and person-centred care: Canadian national position statement on patient-reported outcomes
}

\author{
S. Ahmed, ${ }^{* a}$ L. Barbera MD, ${ }^{\dagger}$ S.J. Bartlett $\mathrm{PhD}^{*}$ D.G. Bebb $\mathrm{BMBCh} \mathrm{PhD}_{1}^{\dagger}$ M. Brundage $\mathrm{MD}_{1}^{\neq}$ \\ S. Bryan PhD, ${ }^{\S}$ W.Y. Cheung MD MPH, ${ }^{+}$N. Coburn MD MPH, ${ }^{\ddagger}$ T. Crump $\mathrm{PhD}_{1}^{+}$ \\ L. Cuthbertson BHSc(OT) MEd PMP, ${ }^{\S}$ D. Howell RN PhD, ${ }^{\ddagger}$ A.F. Klassen BA DPhil(Oxon), ${ }^{\ddagger}$ S. Leduc RN, \\ M. Li MD PhD, ${ }^{\ddagger}$ N.E. Mayo BSc(PT) MSc PhD, ${ }^{*}$ G. McKinnon MD, ${ }^{\dagger}$ R. Olson MD MSc, ${ }^{\S}$ \\ J. Pink PhD RPsych, ${ }^{+}$J.W. Robinson PhD RPsych, ${ }^{+}$M.J. Santana MPharm PhD, ${ }^{\dagger}$ R. Sawatzky PhD RN, ${ }^{\S}$ \\ R.S. Moxam MSc, ${ }^{\ddagger}$ S. Sinclair $\mathrm{PhD}_{,}^{\dagger}$ F. Servidio-Italiano HonBSc BEd MA, ${ }^{\ddagger}$ and W. Temple MD ${ }^{\dagger}$
}

\begin{abstract}
Background Patient-reported outcomes (PROs) are essential to capture the patient's perspective and to influence care. Although PROs and PRO measures are known to have many important benefits, they are not consistently being used and there is there no Canadian PROs oversight. The Position Statement presented here is the first step toward supporting the implementation of PROs in the Canadian health care setting.

Methods The Canadian PROs National Steering Committee drafted position statements, which were submitted for stakeholder feedback before, during, and after the first National Canadian Patient Reported Outcomes (CanPROs) scientific conference, 14-15 November 2019 in Calgary, Alberta. In addition to the stakeholder feedback cycle, a patient advocate group submitted a section to capture the patient voice.
\end{abstract}

Results The CanPros Position Statement is an outcome of the 2019 CanPROs scientific conference, with an oncology focus. The Position Statement is categorized into 6 sections covering 4 theme areas: Patient and Families, Health Policy, Clinical Implementation, and Research. The patient voice perfectly mirrors the recommendations that the experts reached by consensus and provides an overriding impetus for the use of PROs in health care.

Conclusions Although our vision of PROs transforming the health care system to be more patient-centred is still aspirational, the Position Statement presented here takes a first step toward providing recommendations in key areas to align Canadian efforts. The Position Statement is directed toward a health policy audience; future iterations will target other audiences, including researchers, clinicians, and patients. Our intent is that future versions will broaden the focus to include chronic diseases beyond cancer.

Key Words Patient-reported outcomes, patient-reported outcome measures, PROs, PROMs, Canada, patient experiences, implementation science, health policy

Curr Oncol. 2020 April:27(2)90-99

www.current-oncology.com

\section{BACKGROUND AND CONTEXT}

Quality-of-care measurement in oncology has seen a growing shift from an exclusive focus on health outcomes such as toxicity and survival as reported by clinicians, toward the inclusion of outcomes prioritized and reported by patients themselves ${ }^{1}$. Patient-reported outcomes (PROs) are data that are "directly reported by the patient without interpretation of the patient's response by a clinician or

The members of the Canadian Patient-Reported Outcomes National Steering Committee are listed in alphabetic order. 
anyone else and pertains to the patient's health, quality of life, or functional status associated with health care or treatment" 2 . Patient-reported outcomes originate from patients and provide self-reported information on aspects of their health that are relevant to their quality of life, including their symptoms; functioning; and physical, mental, and social health. That information can be used by health care providers, health care organizations, and the health system or policymakers to assess the effect of a medical condition and its treatment from the patient's perspective ${ }^{3}$. In contrast, PRO measures (also known as PROMs) are instruments (surveys, questionnaires, or other self-appraisal tools) that are completed by patients to capture the information ${ }^{4}$.

Evidence demonstrates that PROs benefit many sectors of the health care system. They are an essential part of person-centred care and can improve the quality of patient care $^{5}$, improve patient-clinician communication ${ }^{6-13}$, reduce health services use and emergency department visits ${ }^{8,14}$, assist with problem detection ${ }^{15,16}$, and influence patient management ${ }^{12}$. Importantly, PROs improve patient outcomes such as symptom control, satisfaction ${ }^{6,7}$, health-related quality of life ${ }^{8}$, and functioning ${ }^{10,17-19}$, and have been associated with increased survival duration ${ }^{20-23}$. Collection of PROs provides evidence in the form of patient-centred data for clinicians, payers, researchers, and policymakers, and can assist in the evaluation and implementation of new care methods ${ }^{2}$. In Canada and other jurisdictions, PRO data are a common requisite for payer reimbursement submissions. Effective use of PROs for personalized patient management can help to achieve sought-after "Triple Aim" health care reform goals of better population health, improved patient experience, and lower health care costs ${ }^{4,24-26}$.

Globally, PROs have been used in clinical trials and in many organizations for decades. In Canada, although many research and patient-care organizations are working to incorporate PROs into their clinical processes, the use and implementation of PROs is regionally or provincially siloed and can be fragmented across various medical disciplines ${ }^{21}$. There is no nationally mandated guidance on PROs, and there are no implementation best practices. The uptake and embedding of PROs in "real-world" clinical practice, where the data are used to guide health care decision-making and patient management, are still limited ${ }^{27-30}$. Barriers to the implementation of PROs are well documented ${ }^{3,5,31-35}$.

Our vision is that PROs will transform the health care system to be more patient-centred. The pillars of that transformation include the routine use of PROs in clinical care, advancement of knowledge from continuing research in the field, and the use of aggregate Canada-wide PROs data to enhance care through quality improvement and population-based research.

The Canadian PROs National Steering Committee is a collective national body of stakeholders, including PROs experts inside and outside oncology, pediatrics, and surgery; allied health providers (nursing, social work, psychology); patient advocates; and health care organizations such as the provincial cancer agencies and the Canadian Partnership Against Cancer. The National Steering Committee developed the Position Statement presented here with the intention of transforming the health care system by supporting the integration and implementation of PROs into the Canadian health care setting and by advancing PROs knowledge through research. The Position Statement is intended as a first step toward the national alignment of stakeholders (health care providers, administrators, health policymakers, patient advocacy groups, researchers, and industry) who support the greater use of PROs in Canadian health care and research contexts. This first iteration of the Canadian PROs Position Statement is intended to guide health care decision-makers and leaders with recommendations in 4 areas: Patient and Family, Health Policy, Clinical Implementation, and Research. Included are several overarching position statements and a patient perspective from the Colorectal Cancer Resource and Action Network.

\section{METHODS}

This Position Statement supporting the implementation of PROs in the Canadian health care setting represents a truly collective effort between experts and various stakeholders.

The Position Statement was first developed by a National Steering Committee of PROs experts, with subcommittees-each having a designated section leader-representing each of the 4 themes. Subsequently, the subcommittees identified the priority issues specific to their subtheme, formulated consensus questions, examined evidence, and drafted recommendation statements using available evidence. Although originally intended to be a Consensus Statement, the work product became a Position Statement because the available evidence was insufficient for the recommendations to be entirely evidence-based. In some areas, the recommendations are conceptual or are still based on professional opinion, expertise, or common sense. The recommended position statements for each theme were then presented to the steering committee. Theme leaders, steering committee, and subcommittee members are all listed as authors.

The final Position Statement and the present manuscript arose from the CanPROs scientific conference held 14-15 November 2019 in Calgary, Alberta. Stakeholder feedback on the draft version was obtained from health administration, patient groups, and industry at an in-person stakeholder meeting on 13 November 2019. Wider stakeholder feedback was obtained at the CanPRos scientific conference, where real-time voting by 150 delegates from across Canada was used to gauge support for the position statements. The participating delegates included PROs experts, oncologists, oncology pharmacists and nurses, psychosocial oncology clinicians, cancer care administrators, health policy members, members of patient advocacy groups, and industry representatives. After the conference, the position statements were sent to all CanPROs attendees and the authors for further feedback.

\section{RESULTS: CANADIAN NATIONAL POSITION STATEMENT ON PROS}

\section{Overarching PROs Position Statements}

The Steering Committee identified 3 overarching PROs position statements.

Dedicated resources (including human, financial, health systems) should be invested to integrate PROs 
into clinical care, given their demonstrated value and benefits.

A Canadian national PROs body consisting of PROs experts is needed to guide expert direction in all areas of health care, policy, and research.

- This PROs body would provide direction to national and regional authorities including, for example, Health Canada and provincial, regional, or hospital authorities and government decision-makers such as the Canadian Agency for Drugs and Technologies in Health, the pan-Canadian Oncology Drug Review, and the Institut national d'excellence en santé et services sociaux.

- Responsibilities would include establishing Canadian PROs standards to guide global clinical trials and the appropriate selection of PROMs and interpretation of PROs data for action and decision-making.

The application of PROs must incorporate specific tools and strategies as needed to address equity, diversity, and inclusion. The tools and strategies have to be meaningful, accessible, and useable by all patients, including patients who are affected by differences in ability, language, culture, gender, sex, sexual orientation, socioeconomic status, or place of residence. They have to address the unique needs of diverse and underrepresented groups including Indigenous, Inuit, and Métis individuals.

\section{Position statement on PROs for Patients and Families \\ Section leaders: Susan J. Bartlett PhD and Jennifer C. Pink PhD}

Patient and family-centred cancer care balances the knowledge that the patient is at the centre of care with a growing awareness that illness affects the family ${ }^{36}$. In the position statement presented here, the term "family" refers to the patient's psychological family, including family members, friends, neighbours, and co-workers ${ }^{37}$. "Caregiver," a term frequently used in the PROs literature, excludes family who are profoundly affected by the patient's illness (including children) and who do not directly provide care to the patient. Further, it inadequately captures the nature of the relationship between a patient and the provider of care (for example, spouses of patients ${ }^{38}$ ). Alternative terms for "family" or "caregiver" were suggested, including people within a patient's "circle of care," "the tribe," "the support team."

An emerging area, family-reported outcomes (FROs) measure the effect of an illness on the outcomes of family members themselves ${ }^{39}$. Although FROs are included alongside PROs in the position statement presented here, we acknowledge that involving families might require consent of the patient. Research is needed to identify optimal ways to approach families, the PROs and FROs that can best inform patient- and family-centred care, and how to incorporate the needs, preferences, and values of patients and family members, especially when those needs, preferences, and values might diverge. Additional issues relevant to the implementation of PROs and FROs include burden, privacy, registration, documentation, regulation, and clinical responses and resources, including capacity to respond to patient and family distress (for example, low mood).

Patient- and family-centred care has been widely used (and researched) in cancer care, which was the context of the CanPROs meeting, 14-15 November 2019. A patient- and family-centred approach to care is relevant to other chronic illnesses and can vary with the condition and the wishes of the patient.

A key point is that, as with any person-reported outcome, it is important to use the information collected to inform conversations, shared decision-making, or clinical actions ${ }^{40}$. Using the information is especially important when any level of emotional distress is identified.

Conceptual shift from patient to family as the unit of care

- Because cancer is a family illness, a conceptual shift is needed, whereby care moves from patients only to also include families (where family is defined broadly as the circle of care).

- An essential component of patient- and familycentred care could include the systematic integration of family-reported outcomes (FROs) into cancer care.

Patient consent: ethics and confidentiality

- Important areas that need clarification include the consent of patients to contact families and to control what information (from PROs and FROs) is disclosed to whom.

Patient and family data must be used in real time to enhance care

- PROs and FROs should be used in real time to help identify care gaps and address unmet needs.

- There must be a clinical response each and every time PROs or FROs are collected.

"What I want to do for all the people like me"

- Patients, families, and health care providers need education and training to understand how PROs and FROs can benefit an individual's care, the care of other patients, and the health care system.

"Nothing about me without me"

- Patients and families should co-create PROs and FROs research, including identifying patient- and family-centred research agendas and creating guidelines for the use of existing tools and the development of new tools for measuring PROs and FROs.

\section{Position statement on PROs for Health Policy \\ Section leaders: Richard Sawatzky RN PhD and Stirling Bryan PhD}

The greater demand for measurement and monitoring of PROs by regulators, payers, accreditors, and professional organizations will help to drive implementation of PROs into clinical care ${ }^{5}$. A key point emphasized by this statement is the strong need for a national PROs advisory body to help with the implementation, described in the second overarching position statement (need for a Canadian national PROs body) and again in the third PROs for health policy position statement [implementing such a body (next subsection)]. 
Access to care

- Decision-making about access to care (including current and new technologies, drugs, or therapies) should be guided by a deliberative approach that incorporates PROs. Deliberative frameworks involve the application of multiple criteria in a decision-making process (for example, cost, clinical outcomes, patient experience, social determinants). In such frameworks, PROs should represent a key criterion.

- The selection and use of PROMs must reflect societal and patient perspectives alike. Societal perspectives reflect the values of the general population and are appropriate to guide health policy decisions. However, "patient perspectives" (that is, descriptive information about outcomes and values of those experiencing health challenges and services) must also be considered to ensure that the voices of patients also inform access decisions.

Implementation recommendations

- Establish both national and provincial or territorial bodies to guide selection of PRO measures and interpretation of PRO data for action and decision-making.

System performance

- PROs must be routinely collected and integrated to support continuous learning, improvement, and patient-centred decision-making in health care delivery. That routine collection and integration can be operationalized through a learning health system model in which every patient and every encounter provides an opportunity for PRO data collection to support improvement initiatives ${ }^{41}$.

Implementation recommendations

- Create a national repository of anonymized data provided by validated PROs tools for health care quality improvement.

- Integrate the science, application, and use of PROs into the core health care curricula.

- Include the implementation and adoption of PROs as a mandatory requirement of a health care institution or facility accreditation.

\section{Position Statement on PROs for Clinical Implementation}

Section leader: Doris Howell RN PhD FAAN

A key concept with regard to the implementation of PROs in the clinical context is the difference between dissemination and implementation. Implementation is defined as the use of a specific set of activities and strategies that promote the adoption, integration, and scale-up of evidence-based interventions and innovations in practice within specific settings ${ }^{42}$. Implementation of PROs requires use of best-practice implementation strategies to facilitate the embedding of PROs into routine clinical care ${ }^{43,44}$. As with any change in clinical processes, successful implementation of PROs requires institutional commitment from leadership, buy-in from providers and administrators (and ultimately patients), and coordination of those multiple stakeholders to reach the desired vision for use of PROs in person-centred patient management ${ }^{34}$.
Electronic methods of capturing PROs (including tablet or smartphone apps, dedicated devices, or a Web-based portal) are fundamental to widespread use and scaleup $^{5,8,20,45}$. The PROs data have to interface with electronic health records and be immediately accessible for use in the clinical encounter. Ideally, electronic methods could be used to capture PROs between clinic visits. Access issues such as computer literacy must be acknowledged, because not everyone can engage well with electronic systems. The lack of resources in some areas will prohibit electronic capture methods.

The PROs data must be made available and accessible to inform those for whom it is most relevant: patients and health care professionals. The PROs output and communication materials for patients and physicians have to be accessible across the health literacy spectrum, intuitive, and visually appealing ${ }^{43,46}$.

Finally, a key point is that everyone needs PROs education, including patients, families, and health care providers. Patients and families need education so that they can use PROs in health self-management ${ }^{43}$. Health care providers need training in how to interpret PRO scores, how to incorporate scores into clinical encounters, and how to use the data to inform treatment plans and to communicate with patients in determining "what matters most" and what should be addressed as a priority ${ }^{9,47}$.

Select relevant PROs.

- Engage with patients as partners from the start, together with family advisors and clinicians to select disease-specific PROMs that are meaningful for the patient, clinically relevant, and appropriate for clinical research. A patient-oriented approach has patients participating in all aspects of PROs evaluation, including selection, implementation, research, and evaluation.

- Use robust communication strategies to engage all care team members and the patient from the first point of contact and at strategic time points during care.

- Integrate PROs into the clinical workflow, care pathways, and the way that team members work together so that use of PROs becomes routine practice.

- Capture PROs before and between each clinical encounter to document adverse events and other patient issues, and configure workflow to ensure a response to the data.

- Invest in electronic capture methods for PROs that integrate with patient records. That PROs model represents the ideal.

- Include documentation systems and analytics in PROs programs to enable data abstraction for quality improvement, performance measurement, and research ${ }^{41}$.

Clinicians use PROs in a real-world setting for personcentred care.

- Educate clinicians initially and on a continuing basis about how to interpret PRO scores, to integrate those data into everyday patient management, and to understand the benefits of use $\mathrm{e}^{47}$. Clinicians require specific and ongoing practice 
coaching for integrating PROs data into shared decision-making and treatment planning ${ }^{48}$.

- Create intuitive clinician-facing reports that summarize PRO scores for ease of interpretation and actionability ${ }^{46}$.

- Align PRO scores and evidence in best-practice guidelines that direct clinicians to the selection and application of optimal clinical interventions to address detected problems.

- Include mechanisms for evaluating the implementation of PROs in patient management and for ongoing quality improvement.

PROs support patients in becoming informed partners in achieving better health outcomes.

n Create an inclusive and accessible education program to help patients and families who are first entering the cancer system learn why PROs are collected and how PROs can improve their health outcomes.

- Patients and families need to learn how to interpret PROs scores and score changes, and they need guidance about actions to take and the use of the data for communicating with the health care team.

- Patient and families need to learn that PROs can be used for symptom monitoring, early intervention, informing treatment plans, understanding disease patterns and health recovery, and guiding self-management behaviours.

- Develop visually appealing patient-friendly PROs reports for easy interpretation and an understanding about how PROs changes align with disease and treatment changes.

- Integrate PROs data with patient portals so that patients can link PROs data with clinical and laboratory data, and complete PROs between clinic visits.

\section{Position Statement on PROs for Research} Section leaders: Lisa Barbera MD and Madeline Li MD PhD

Research involving PROs has 5 foci $^{49}$ :

As outcomes in the scientific evaluation of various treatments

As naturalistic data in clinical research

In the development and psychometric analysis of PROMs In implementation science related to the routine integration of PROs into clinical care

In evaluating the effect of PROs use on health systems and patient care

The patient perspective has to be consistently incorporated into research. That effort includes data collection that goes beyond survival to include measures of symptom burden, toxicity, quality of life, and functional status from the patient perspective ${ }^{23}$. The research community ought to provide information to patients, family members, and clinicians that details how various treatment options might affect those important outcomes.

The clinical benefits of using PROs in routine care have been demonstrated for symptom identification, communication, and satisfaction. Further research is required to understand if additional outcomes are affected. Further work is also required to understand how best to implement clinical programs of PRO measurement.

The goal of all PRO applications has to be defined up front, with a research lens applied to all PRO uses.

- The goal-screening, routine clinical care, clinical trial endpoint, or basic research-will determine the nature, frequency, and optimal length of use of the selected PROMs.

- An a priori research lens in all applications (patient and family care, health policy, implementation science) facilitates a project design and evaluation framework for optimal outcome measurement.

Agreement on a minimum common dataset should be established to permit comparative research and to allow for synergies with health policy and other research groups.

- Core domains to evaluate with PROMs should constitute a minimum common dataset.

- Consensus is needed concerning the diseasespecific, phase-specific, and treatment-specific PROMs that should be added to the core measures to balance the capabilities and needs of the system, the patient, and the clinician.

Clinical applications are a priority for PROM research development.

- Computer-adaptive methods can facilitate collection of both core and disease-specific PROMs, and psychometric research about the clinical application of such an approach is needed.

- Implementation science and PROMs development should focus on under-researched populations such as minority or disadvantaged groups and the cancer survivorship space.

Research about PROs should now focus on the effect of PROs on clinically relevant outcomes such as health services use and survival.

- Early evidence for the effects of PROs use on health care utilization and survival has been developed, but there is an existing knowledge gap concerning the mechanisms of those relationships or their cost-effectiveness.

- Although research about the additional effect of PRO measurement is required, reduced symptom burden might not be a clinically plausible outcome for evaluation.

\section{Position Statement on PROs from the Patient Advocate Perspective} Section leader: Filomena Servidio-Italiano

The position statement presented here was developed by the Colorectal Cancer Resource and Action Network. Although specific to patients with colorectal cancer, it intends to be inclusive of the beliefs and perspectives of other cancer patients. It has received endorsement from the Breast Cancer Society of Canada and Ovarian Cancer Canada.

PROs should be captured as study endpoints in the development and evaluation of new therapies, and PROMs should include questions related to survivorship, 
health, and quality of life equally when evaluating new therapies, especially in the advanced-disease setting. PROs can be used for adverse event reporting, and PROMs designed to measure adverse effects of a therapy should be administered in the setting most suitable for the patient, at the time that the adverse effects are most prominent and top of the patient's mind so that the patient perspective is captured more fully.

PROs data collected in the clinic should be reviewed before patient consultation and used to guide and improve clinical care. Health and quality-of-life data have to be captured in addition to treatment-induced side effects that might require supportive care measures. Clinical guidelines have to be in place to guide clinician responses to patient concerns and issues identified in the patient's data during the clinic visit. Consider adding a section on the PROs questionnaire for completion by the family. Such a section could be helpful for both the patient and the care partner who is playing the vital role of caring for the patient. Additional information might be captured on perceived health outcomes, including the patient's health and quality of life, symptoms, daily functioning, and aspects of physical, psychological, and social well-being. Completion of this part of the questionnaire might also identify whether supportive care services are required for the person supporting the patient.

- Standardizing nationally-collected PROs data might help to standardize clinical practice. Currently, there is no consistency from one cancer centre to another in the PROMs that are administered.

- Patient education has to be a PROs component. Patients need to be taught and to acquire familiarity with the various terms used in PROs questionnaires, to understand what rankings or ratings mean, and how the data will be used after the questionnaire is completed. They need to understand how to distinguish treatment-induced adverse events from disease-induced symptoms so that they can complete the questionnaire with greater ease and competency.

\section{DISCUSSION AND CONCLUSIONS}

The Position Statement presented here demonstrates Canadian leadership in PROs and represents the beginning of a national alignment of patients, policymakers, researchers, and health care providers toward greater use and effect of PROs in Canadian health care and research contexts. We were encouraged that the preceding subsection relating to the patient advocate perspective perfectly mirrors the recommendations that experts reached by consensus, which are highlighted in all four of the themes. That alignment reassures us that we are on the right track.

We acknowledge that our vision, in which PROs transform the health care system to be more patient-centred and integrated into every health care encounter, is aspirational and will not be easily achieved in the short term. We also acknowledge that some important aspects are not yet adequately addressed, including many concerns with respect to privacy (data transparency and access, permissions, sharing, and reporting), inclusion and diversity, health literacy, and access. Importantly, PROs do not currently fully embrace diversity, and there are significant issues with certain groups not being represented or, worse, being misrepresented. Those areas suggest topics for future research. We recognize that to make changes and improvements in the health system, we have to articulate where we are and then move forward.

Collaboration is a key value as we proceed. Various stakeholder groups all have different needs and priorities surrounding the use of PRO data; however, there could be synergy in selecting PROs data and PROMs that are used across all the pillars of patients and families, health policy, clinical implementation, and research. Aligning outcomes will reduce burden on the system. Our intention is that future versions of the Position Statement will broaden the focus to include chronic diseases beyond cancer. The release of the next iteration of the PROs Position Statement will coincide with the second PROs conference, hopefully to be held in 2021. We invite you to choose to engage and participate.

\section{ACKNOWLEDGMENTS}

The following organizations provided funding for the CanPROs meeting held 14-15 November 2019 in Calgary, Alberta, and for the creation of this manuscript: Pfizer Canada; AbbVie Canada; Amgen; AstraZeneca; Bristol-Myers Squibb; Bayer Healthcare; Taiho Oncology; Albertans 4 Health Research; Alberta SPOR SUPPORT Unit; and Department of Oncology, University of Calgary.

The creation of the Position Statement was a collective effort. The authors thank the many patient and family activists and stakeholders who were involved; the Position Statement is a result of their commitment and contributions. The authors acknowledge Alberta Health Services, the University of Calgary, BC Patient-Centred Measurement, the Southern Alberta Institute of Urology, and Trinity Western University School of Nursing. Finally, the authors thank Chrystal Palaty PhD, Metaphase Health Research Consulting Inc., for her support in preparing the final manuscript for submission.

\section{CONFLICT OF INTEREST DISCLOSURES}

We have read and understood Current Oncology's policy on disclosing conflicts of interest, and with the exceptions of the authors listed below, we declare that we have none or were unable to respond to the request for disclosures because of COVID-19related activities. LB has received an honorarium from Genentech and travel support from Elekta. AFK has received consulting fees from Allergan. RO has received grant funding from Varian Medical Systems.

\section{AUTHOR AFFILIATIONS}

*Quebec: Department of Medicine School of Physical and Occupational Therapy, McGill University, Montreal (Ahmed, Mayo); Faculty of Medicine, McGill University, and McGill University Health Systems, Montreal (Bartlett); ${ }^{\dagger}$ Alberta: Department of Oncology, University of Calgary, Calgary (Barbera, Bebb, Cheung); University of Calgary, Calgary (Crump, Temple); Departments of Surgery and Oncology, University of Calgary, Calgary (McKinnon); Department of Oncology, Cumming School of Medicine, University of Calgary, Calgary (Pink, Santana); University of Calgary and Tom Baker Cancer Centre, Calgary (Robinson); Faculty of Nursing, University of Calgary, Calgary (Sinclair); ${ }^{\ddagger}$ Ontario: Queen’s Cancer Research Institute, Kingston (Brundage); Sunnybrook Health Sciences Centre, Toronto (Coburn); Lawrence S. Bloomberg Faculty of Nursing, University of Toronto, Toronto (Howell); McMaster University, Hamilton (Klassen); CancerInsight, Oakville (Leduc); Department of Supportive Care, Princess Margaret Cancer Centre, 
University of Toronto, Toronto (Li); Canadian Partnership Against Cancer, Toronto (Moxam); Colorectal Cancer Resource and Action Network, Oakville (Servidio-Italiano); ${ }^{\S}$ British Columbia: School of Population and Public Health, University of British Columbia, Vancouver (Bryan); Office of Patient-Centred Measurement, British Columbia Ministry of Health, Vancouver (Cuthbertson); Radiation Oncology and Developmental Radiotherapeutics, University of British Columbia, Prince George (Olson); School of Nursing, Trinity Western University, Langley (Sawatzky).

\section{REFERENCES}

1. LeBlanc TW, Abernethy AP. Patient-reported outcomes in cancer care-hearing the patient voice at greater volume. Nat Rev Clin Oncol 2017;14:763-72.

2. Weldring T, Smith SMS. Patient-reported outcomes (PROs) and patient-reported outcome measures (PROMs). Health Serv Insights 2013;6:61-8.

3. Mercieca-Bebber R, King MT, Calvert MJ, Stockler MR, Friedlander M. The importance of patient-reported outcomes in clinical trials and strategies for future optimization. Patient Relat Outcome Meas 2018;9:353-67.

4. Canadian Institute for Health Information (CIHI). PROMs Background Document. Ottawa, ON: CIHI; 2015.

5. Jensen RE, Rothrock NE, DeWitt EM, et al. The role of technical advances in the adoption and integration of patientreported outcomes in clinical care. Med Care 2015;53:153-9.

6. Chen J, Ou L, Hollis SJ. A systematic review of the impact of routine collection of patient reported outcome measures on patients, providers and health organisations in an oncologic setting. BMC Health Serv Res 2013;13:211.

7. Kotronoulas G, Kearney N, Maguire R, et al. What is the value of the routine use of patient-reported outcome measures toward improvement of patient outcomes, processes of care, and health service outcomes in cancer care? A systematic review of controlled trials. J Clin Oncol 2014;32:1480-501.

8. Basch E. Patient-reported outcomes-harnessing patients' voices to improve clinical care. N EnglJ Med 2017;376:105-8.

9. Yang LY, Manhas DS, Howard AF, Olson RA. Patient-reported outcome use in oncology: a systematic review of the impact on patient-clinician communication. Support Care Cancer 2018;26:41-60.

10. Velikova G, Booth L, Smith AB, et al. Measuring quality of life in routine oncology practice improves communication and patient well-being: a randomized controlled trial. J Clin Oncol 2004;22:714-24.

11. Berry DL, Blumenstein BA, Halpenny B, et al. Enhancing patient-provider communication with the electronic selfreport assessment for cancer: a randomized trial.JClin Oncol 2011;29:1029-35.

12. Santana MJ, Feeny D, Johnson JA, et al. Assessing the use of health-related quality of life measures in the routine clinical care of lung-transplant patients. Qual Life Res 2010;19:371-9.

13. Detmar SB, Muller MJ, Schornagel JH, Wever LD, Aaronson NK. Health-related quality-of-life assessments and patientphysician communication: a randomized controlled trial. JAMA 2002;288:3027-34.

14. Howell D, Li M, Sutradhar R, et al. Integration of patientreported outcomes (PROs) for personalized symptom management in "real-world" oncology practices: a population-based cohort comparison study of impact on healthcare utilization. Support Care Cancer 2020;: [In press].

15. Marshall S, Haywood K, Fitzpatrick R. Impact of patientreported outcome measures on routine practice: a structured review. J Eval Clin Pract 2006;12:559-68.

16. Haywood K, Marshall S, Fitzpatrick R. Patient participation in the consultation process: a structured review of intervention strategies. Patient Educ Couns 2006;63:12-23.
17. Cleeland CS, Wang XS, Shi Q, et al. Automated symptom alerts reduce postoperative symptom severity after cancer surgery: a randomized controlled clinical trial. J Clin Oncol 2011;29:994-1000.

18. McLachlan SA, Allenby A, Matthews J, et al. Randomized trial of coordinated psychosocial interventions based on patient self-assessments versus standard care to improve the psychosocial functioning of patients with cancer. J Clin Oncol 2001;19:4117-25.

19. Basch E, Deal AM, Kris MG, et al. Symptom monitoring with patient-reported outcomes during routine cancer treatment: a randomized controlled trial. J Clin Oncol 2016;34:557-65.

20. Basch E, Deal AM, Dueck AC, et al. Overall survival results of a trial assessing patient-reported outcomes for symptom monitoring during routine cancer treatment. JAMA 2017; 318:197-8.

21. Olson RA, Howard F, Lapointe V, et al. Provincial development of a patient-reported outcome initiative to guide patient care, quality improvement, and research. Healthc Manage Forum 2018;31:13-17.

22. Sperti E, Di Maio M. Outcomes research: integrating PROs into the clinic-overall survival benefit or not, it's worth the trouble. Nat Rev Clin Oncol 2017;14:529-30.

23. Rivera SC, Kyte DG, Aiyegbusi OL, Slade AL, McMullan C, Calvert MJ. The impact of patient-reported outcome (PRO) data from clinical trials: a systematic review and critical analysis. Health Qual Life Outcomes 2019;17:156.

24. Dew T, Huebner ES. Adolescents' perceived quality of life: an exploratory investigation. J Sch Psychol 1994;32:185-99.

25. Howell D, Liu G. Can routine collection of patient reported outcome data actually improve person-centered health? Healthc Pap 2011;11:42-7.

26. Berwick DM, Nolan TW, Whittington J. The Triple Aim: care, health, and cost. Health Aff (Millwood) 2008;27:759-69.

27. Stover AM, Basch EM. Using patient-reported outcome measures as quality indicators in routine cancer care. Cancer 2016;122:355-7.

28. Anatchkova M, Donelson SM, Skalicky AM, McHorney $C A$, Jagun D, Whiteley J. Exploring the implementation of patient-reported outcome measures in cancer care: need for more real-world evidence results in the peer reviewed literature. J Patient Rep Outcomes 2018;2:64.

29. Gonçalves Bradley DC, Gibbons C, Ricci-Cabello I, et al. Routine provision of information on patient-reported outcome measures to healthcare providers and patients in clinical practice. Cochrane Database Syst Rev 2015;:CD011589.

30. Seow H, Sussman J, Martelli-Reid L, Pond G, Bainbridge D. Do high symptom scores trigger clinical actions? An audit after implementing electronic symptom screening. J Oncol Practice 2012;8:e142-8.

31. Howell D, Molloy S, Wilkinson K, et al. Patient-reported outcomes in routine cancer clinical practice: a scoping review of use, impact on health outcomes, and implementation factors. Ann Oncol 2015;26:1846-58.

32. Porter I, Goncalves-Bradley D, Ricci-Cabello I, et al. Framework and guidance for implementing patient-reported outcomes in clinical practice: evidence, challenges and opportunities. J Comp Eff Res 2016;5:507-19.

33. Howell D, Hack TF, Green E, Fitch M. Cancer distress screening data: translating knowledge into clinical action for a quality response. Palliat Support Care 2014;12:39-51.

34. Basch E, Snyder C. Overcoming barriers to integrating patient-reported outcomes in clinical practice and electronic health records. Ann Oncol 2017;28:2332-3.

35. Warsame R, D'Souza A. Patient reported outcomes have arrived: a practical overview for clinicians in using patient reported outcomes in oncology. Mayo Clin Proc 2019;94:2291-301. 
36. Basra MK, Finlay AY. The family impact of skin diseases: the Greater Patient concept. Br J Dermatol 2007;156:929-37.

37. Boss P, Dahl CM: Family therapy for the unresolved grief of ambiguous loss. In: Kissane DW, Parnes F, eds. Bereavement Care for Families. New York, NY: Routledge; 2014: 171-82.

38. Molyneaux V, Butchard S, Simpson J, Murray C. Reconsidering the term "carer": a critique of the universal adoption of the term "carer". Ageing Soc 2011;31:422-37.

39. Loiselle CG, Howell D, Nicoll I, Fitch M. Toward the development of a comprehensive cancer experience measurement framework. Support Care Cancer 2019;27:2579-89.

40. Howell DA, Bultz BD, Fitch MI, et al. Ensuring a high-quality response to screening for distress data: systematic knowledge translation is needed to improve patient experience. Oncology Exchange 2012;11:e35-41.

41. Basch E, Spertus J, Dudley RA, et al. Methods for developing patient-reported outcome-based performance measures (PRO-PMs). Value Health 2015;18:493-504.

42. Eccles MP, Mittman BS. Welcome to Implementation Science [editorial]. Implement Sci 2006;1:1.

43. Aaronson N, Elliott T, Greenhalgh J, et al. User's Guide to Implementing Patient-Reported Outcomes Assessment in Clinical Practice. Ver. 2. Milwaukee, WI: International Society for QualityofLifeResearch;2015. [Availableonlineat:https://www.
isoqol.org/wp-content/uploads/2019/09/2015UsersGuideVersion2.pdf; cited 23 December 2019]

44. Basch E, Barbera L, Kerrigan CL, Velikova G. Implementation of patient-reported outcomes in routine medical care. Am Soc Clin Oncol Educ Book 2018;38:122-34.

45. Bennett AV, Jensen RE, Basch E. Electronic patient-reported outcome systems in oncology clinical practice. CA Cancer J Clin 2012;62:337-47.

46. Snyder C, Smith K, Holzner B, Rivera YM, Bantug E, Brundage M on behalf of the PRO Data Presentation Delphi Panel. Making a picture worth a thousand numbers: recommendations for graphically displaying patient-reported outcomes data. Qual Life Res 2019;28:345-56.

47. Santana MJ, Haverman L, Absolom K, etal. Training clinicians in how to use patient-reported outcome measures in routine clinical practice. Qual Life Res 2015;24:1707-18.

48. Damman OC, Jani A, de Jong BA, et al. The use of Proms and shared decision-making in medical encounters with patients: an opportunity to deliver value-based health care to patients. J Eval Clin Pract 2020;26:524-40.

49. Mooney K, Berry DL, Whisenant M, Sjoberg D. Improving cancer care through the patient experience: how to use patient-reported outcomes in clinical practice. Am Soc Clin Oncol Educ Book 2017;37:695-704. 